\title{
EEG Correlates of Spatial Orientation in the Human Retrosplenial
}

\section{Complex}

\author{
Lin, C.-T. ${ }^{1,2,3}$, Chiu, T.-C. ${ }^{1}{ }_{2}^{2}$, and Gramann, K. ${ }^{4,5}$ \\ ${ }^{1}$ Brain Research Center, University System of Taiwan, Hsinchu, Taiwan; \\ 2Department of Computer Science, National Chiao-Tung University, Hsinchu, Taiwan; \\ ${ }^{3}$ Faculty of Engineering and Information Technology, University of Technology, Sydney, Australia \\ ${ }_{4}^{4}$ Biological Psychology and Neuroergonomics, Technische Universitaet Berlin; \\ ${ }_{5}$ Center for Advanced Neurological Engineering, University of California, San Diego, USA
}

\section{Abstract}

Studies on spatial navigation reliably demonstrate that the retrosplenial complex (RSC) plays a pivotal role for allocentric spatial information processing by providing heading information and by translating spatial information represented based on egocentric and allocentric spatial reference frames (SRF). While more and more imaging studies investigate the role of the RSC in spatial tasks, high temporal resolution measures such as electroencephalography (EEG) are missing. To investigate the function of the RSC in spatial navigation with high temporal resolution we used EEG to analyze spectral perturbations during navigation based on allocentric and egocentric SRF. Participants performed a path integration task in a clearly structured virtual environment providing allothetic information. Continuous EEG recordings were decomposed by independent component analysis (ICA) with subsequent source reconstruction of independent time source series using equivalent dipole modeling. Time-frequency transformation was used to investigate reference frame-specific orientation processes during navigation as compared to a control condition with identical visual input but no orientation task. Our results demonstrate that navigation based on an egocentric reference frame recruited a network including the parietal, motor, and occipital cortices with dominant perturbations in the alpha band and theta modulation in frontal cortex. Allocentric navigation was accompanied by performancerelated desynchronization of the 8-13 Hz frequency band and synchronization in the 12$14 \mathrm{~Hz}$ band in the RSC. The results support the claim that the retrosplenial cortex is central to translating egocentric spatial information into allocentric reference frames. Modulations in different frequencies with different time courses in the RSC further provide first evidence of two distinct neural processes reflecting translation of spatial information based on distinct reference frames and the computation of heading changes. 


\section{Introduction}

3 Spatial navigation is central for the survival of a species and requires integration of allothetic information (e.g., landmarks) as well as idiothetic information (e.g., vestibular input) to construct spatial representations. The resulting representations can be broadly classified to be based on allocentric or egocentric spatial reference frames (SRFs) with the origin of the underlying coordinate system centered on aspects of the environment or the ego, respectively (Klatzky, 1998). An allocentric representational system is an object- or environment-centered system. Such a system represents the location of entities (including the navigator) with respect to allothetic information like cardinal directions, the geometry of an environment, or geographical slant (Restat et al., 2004, Kelly et al., 2008). In contrast, the egocentric representational system is centered on the cognizing subject. Thus, the description of object locations based on an egocentric representation depends on the position and orientation of the observer and changes according to navigators' movements (Klatzky, 1998).

Imaging studies investigating the neural structures underlying distinct SRFs demonstrate a central role of medial temporal brain structures for allocentric SRFs (Maguire et al., 1998; Ekstrom et al., 2003; Wolbers and Büchel, 2005; Doeller et al., 2010; Jacobs et al., 2013; Howard et al., 2014). The use of an egocentric SRF, in contrast primarily involves the parietal cortex which integrates self-motion cues from the visual, vestibular, and kinesthetic systems (Bremmer et al., 2001; Cohen and Andersen, 2002; Seubert et al., 2008). The retrosplenial complex (RSC), comprising the caudal cingulate cortex (BA 29 and 30) as well as part of the medial parietal cortex (BA 23 and 31), plays an important role in the exchange and integration of egocentric and allocentric information (Byrne et al., 2007; Vann et al., 2009; Zhang et al., 2012; Dhindsa et al., 2014) and in computing heading directions based on local landmarks (Marchette et al., 2014). Recently, our group demonstrated modulations of brain dynamics in the RSC accompanying the use of an allocentric SRF (Gramann et al., 2010; Plank et al., 2010). Power modulations in the alpha $(8-13 \mathrm{~Hz})$ frequency range in the RSC correlated with homing accuracy only for allocentric navigators (Chiu et al., 2012). Given the behavioral differences dependent on individual SRF proclivities, brain dynamics accompanying use of distinct SRFs might indicate more general differences in individual spatial abilities (Just and Carpenter, 1985; Gramann, 2013). 
Egocentric and allocentric spatial representations entail different information that might be of varying relevance when navigating different environments (Touretzky and Redish, 1996; Burgess, 2006; Gramann, 2013). However, individuals differ with respect to their preference to use a specific SRF or a subset of SRFs during navigation (Gramann et al., 2005, 2012; Bohbot et al., 2007; Riecke and Wiener, 2007; Riecke, 2008; Iglói et al., 2009; Goeke et al., 2013; Gramann, 2013). These individual reference frame proclivities can be observed even when the preferred reference frame is inadequate for a given situation (Iaria et al., 2003).

The present study analyzed individual SRF proclivities during navigation and how these impact brain dynamics in navigationally relevant brain regions including the RSC. In our previous studies we presented sparse visual flow without landmarks. As a consequence of the absence of clear allothetic information, some navigators might have demonstrated a proclivity for an egocentric SRF. In contrast, other studies providing allothetic information (Maguire et al., 1998; Janzen and van Turennout, 2004; Wolbers et al., 2004; Bohbot et al., 2007) did not control for differences in the preferred use of distinct SRFs. The present study addressed individual differences in brain dynamics accompanying use of egocentric and allocentric SFRs during navigation in a highly structured environment including allothetic information. Participants performed a path integration task in an open maze-like environment while the electroencephalogram (EEG) was recorded and subsequently analyzed using adaptive mixture independent component analysis (AMICA) to test how EEG activity was associated with reference frame-specific orientation performance.

\section{Materials and Methods}

\section{Participants}

Twenty-one right-handed male participants were paid for the experiment (mean age = 21 years, $s d=1.20$ years). None of the participants had a history of neurological or psychiatric disorders or a history of drug or alcohol abuse. All participants reported normal or corrected to normal vision and sufficient sleep the night before performing the experiment. They were not aware of the hypotheses at the time of testing and all participants gave their written informed consent to the procedure before the experiment. 


\section{Experimental Paradigm}

2 Participants performed a 2D virtual reality path integration task while sitting comfortably in a dimly room with a viewing distance of $60 \mathrm{~cm}$ from a 24" LCD screen.

In each trial, participants saw visual flow indicating forward movements in space including straight segments before and after one stimulus turn to the left or right. Different path layouts included 1, 2 or 3 straight segments before the stimulus turn and 1,2 , or 3 straight segments after the stimulus turn. The starting position was kept constant and 18 different goal positions could be reached by combining different numbers of straight segments before and after the stimulus turn (e.g., in figure 1A, goal position "a" was reached by combining one straight segment before and one straight segment after the stimulus turn while end position " $h$ " was realized by a combination of three straight segments before and two straight segments after the stimulus turn). Each of the possible combinations was repeated 8 times. Movement speed was adjusted to provide a smooth transition with a general navigation speed of $0.4 \mathrm{~m} / \mathrm{s}$. Before each stimulus turn movement slowed down until the visual flow stopped and subsequently indicated a turn on the spot. All stimulus turns were composed of either a positive or a negative $90^{\circ}$ rotation on the spot. After a turn, the visual flow pattern indicated acceleration to navigation velocity, which was kept constant until the end of the passage where the visual flow pattern again slowed down. The acceleration and deceleration were smooth and limited to a time period of 1.06 sec. Overall, in 144 trials participants were randomly moved to one of 18 possible end positions.

At the end of each path, a 3D homing arrow was displayed (Figure 1B), and participants were required to press the 'left' or 'right' arrow key to indicate whether the start position was either to their 'rear right' or 'rear left' relative to their current position at the end of the passage. This initial selection was used to determine participants' preferred reference frame for each single trial, i.e. whether participants 
1 responded based on an allocentric or an egocentric reference frame (Chiu et al., 2012).

2 Previous studies demonstrated that participants using an egocentric reference frame update their cognitive heading and point back to their left after a visual turn to the left (see Figure 1D). Participants using an allocentric reference frame, in contrast, would respond based on their physical heading to indicate the start position to be located to their rear right after a turn to the left (Gramann et al., 2005). After this initial key press the homing arrow started rotating and participants were instructed to press the down key when the desired homing angle was reached. The second response indicated the individually adjusted homing angle and allowed for computation of angular information like absolute and relative homing errors.

Control Condition. At the beginning of each experimental block, participants first saw a picture of a homing arrow pointing into a random direction (range: $-180^{\circ}$ to $180^{\circ}$ ) for two seconds. Their task was to remember the pointing direction of the arrow and to reproduce this orientation at the end of a control navigation passage. During the control passages, participants saw visual flow patterns indicating movement through the maze along a path composed of random translations and turns for about 1 minute. Participants were asked to attend to the visual flow without actively orienting. At the end of the passage, a 3D arrow was displayed and participants had to adjust the arrow to match the initially presented arrow. This way, participants perceived visual flow during random walks through the environment without the need for spatial updating (Wolbers et al., 2007). Only the last $1 / 3$ of movement during control passages (20 seconds), were kept for further analysis to avoid inclusion of brain dynamics accompanying unintentional spatial updating by the participants in the beginning of the 1-min navigation passage.

Experimental trials. Participants were asked to practice the homing and control trial for at least 5 minutes until they were familiar with the environment and the input device. Subsequently, each participant was required to complete 4 experimental blocks of 36 trials each with every block beginning with a control trial. In addition, each experimental block contained two filler trials with two stimulus turns to avoid participants from building expectations about path configurations and to keep them attending to the task. Between experimental blocks, participants could take a break for about 5-10 minutes. 
2 Strategy categorization and navigation performance. Participants were first classified as Nonturners (using an allocentric reference frame) and Turners (using an egocentric reference frame) according to their initial homing responses (Gramann et al., 2005). To be categorized as Turner or Nonturner, participants had to consistently adjust a strategy-specific homing direction on at least $80 \%$ of all trials. This was the case for all 21 participants (mean strategy-consistent adjustments $=98.4 \%$, $\mathrm{sd}=2.1 \%$ ) resulting in one group of 9 allocentric and one group of 12 egocentric participants. Trials with responses based on the non-preferred strategy were excluded and only trials with strategy-consistent responses were further analyzed. To investigate the difference in task performance between participants using an egocentric or an allocentric reference frame mean homing errors of the subsequent homing adjustments were analyzed. Homing adjustments were defined as the angular adjustment of the homing arrow based on the strategy-specific reference frame. Homing errors were defined as the signed deviation from the expected strategy-specific homing adjustment and thus allowed for investigating over- and underestimation of the correct homing angle. Trials with turns to the left were merged with right-turn trials after trials with homing errors exceeding three times the standard deviation of the individual mean error were removed as outliers.

\section{EEG Recording and Analyses}

Physiological data were recorded using $64 \mathrm{Ag} / \mathrm{AgCl}$ electrodes. All electrodes were placed in an elastic cap according to a subset of a 10\%_-system with impedances below $5 \mathrm{k} \Omega$. EEG data were recorded with Scan NuAmps Express system (Compumedics Ltd., VIC, Australia) referenced to $\mathrm{Cz}$ and digitized at $1 \mathrm{KHz}$ and 32-bit quantization precision.

EEG Analyses. The recorded EEG data were analyzed using the open source toolbox EEGLAB (Delorme and Makeig, 2004) (http://sccn.ucsd.edu/eeglab). EEG data from 64 signals were first down-sampled to $250 \mathrm{~Hz}$ and then filtered to remove frequencies above $50 \mathrm{~Hz}$ and below $0.5 \mathrm{~Hz}$. The filtered data was visually inspected in the channel and the time domain and noisy channels or short time periods with bursts of higher frequencies resembling muscle artifacts were cleaned by manually marking and subsequently removing these from the continuous data. Noisy channels were removed when channel data indicated no activity over longer time periods indicating 'dead 
1 channels' or when the channel data displayed strong deviation from neighboring channels over extended periods of time. On average, this led to the removal of 4.4 channels (3.2 SD) per participant.

After manually cleaning the data, independent component analysis (ICA) was applied to the EEG data in order to extract independent components (ICs) from scalp electrode signals reflecting maximally statistical independent source time series. An adaptive mixture independent component analysis (AMICA) was applied using default parameters with one model (Palmer et al., 2006; Delorme et al., 2012). An equivalent current dipole model was then computed for each IC using DIPFIT2 routines from EEGLAB (Oostenveld and Oostendorp, 2002). ICs with residual variance of less than $15 \%$ for the equivalent dipole model were selected for component clustering. Epochs of straight segments before the turn, the stimulus turn, and straight segments after the turn were extracted from IC-activation time courses of each navigation trial for further time-frequency and correlation analysis.

\section{Time-frequency analysis and event-related spectral perturbation (ERSP) statistics.}

Epochs including straight segments before and after turns as well as segments including a turn were extracted and subsequently ERSP images were computed by converting ICactivation time courses of the extracted epochs to the frequency domain. To this end, a moving window Fast Fourier Transform (FFT) with a window length of 128 points and overlap of 124 points was computed. Because straight segments before and after a turn could be of different length dependent on the final position, the 'timewarp' procedure in EEGLAB was used to linearly transform the ERSP matrices in order to normalize the time length difference between trials. This included linear warping of power values to the mean length of all epochs. Subsequently, mean log power from control trials was subtracted to compute ERSP images showing increases and decreases in spectral power during the task as compared to the control condition (Makeig, 1993). Finally, ERSPimages of all epochs of the trial were concatenated into the grand ERSP time course for the complete navigation phase.

Using bootstrapping, a nonparametric permutation-based statistical test with false discovery rate (FDR) multiple comparison correction for controlling false alarm, timefrequency points during navigation that did not differ significantly from control trial activity were masked and significant $(\mathrm{p}<0.05)$ perturbations were color-coded. 
1 Component clustering and cross-subject analysis. Individual ICs were clustered based on the time course of event-related potentials (ERPs), mean IC log spectra, equivalent dipole locations, event-related spectral perturbation (ERSP), and intertrial coherence (ITC), replicating the setting used in previous studies (Gramann et al., 2010; Chiu et al., 2012). Weighted IC measures were summed and compressed by principal component analysis (PCA) resulting in a 25-dimensional vector. Finally, ICs were clustered by applying the $K$-means algorithm to the 25-dimensional measure clustering the data according to the distance of measures between each other in the vector space. The $K$-means method randomly initials the centroid of clusters and updates centroids by minimizing the distance within and maximizing the distance between clusters ICs with a distance larger than three standard deviations from the final mean of any cluster centroid were marked as outlier and removed from the analysis.

From an initial set of 1,209 ICs of all participants, 897 ICs with a residual variance of the equivalent dipole model of less than 15\% were clustered. Based on the average number of remaining ICs for all participants, the number of resulting clusters returned by k-means was set to 40 clusters. Finally, 8 clusters of interest with a total of 149 ICs that were identified as brain sources based on the dipole model location of the cluster centroids were selected for further analysis. Other clusters reflecting muscle activity and eye-movements or artifacts as well as brain clusters without task-relevant power modulations were not considered further. The selected 8 clusters were located in or near the anterior medial frontal cortex (Cls $1 ; \mathrm{x}=-1, \mathrm{y}=37, \mathrm{z}=33 ; 14$ ICs, 11 participants), the left and right motor cortex (Cls 2: $\mathrm{x}=-41, \mathrm{y}=-16, \mathrm{z}=44 ; 14$ ICs, 12 participants and Cls 3: $\mathrm{x}=40, \mathrm{y}=-19, \mathrm{z}=44 ; 20$ ICs, 18 participants, respectively), the left and right superior parietal cortex (Cls 4: $\mathrm{x}=-17, \mathrm{y}=-37, \mathrm{z}=42 ; 23$ ICs, 17 participants, and Cls 5: $\mathrm{x}=12, \mathrm{y}=-$ $41, z=39 ; 23$ ICs, 19 participants, respectively), the RSC (Cls 6: $x=12, y=-57, z=6 ; 18$ ICs, 12 participants), and the middle occipital cortex (Cls 7: $x=7, y=-87, z=24 ; 23$ ICs, 17 participants), as well as the bilateral occipital cortex (Cls 8: $\mathrm{x}=21, \mathrm{y}=-87, \mathrm{z}=12 ; 14$ ICs, 14 participants).

For cross-subject analysis, all epochs of ICs were concatenated from a cluster across subjects after averaging multiple ICs of individual participants contributing to the same cluster. Subsequently ERSPs were computed on the concatenated data to further display the average ERSP of a component cluster (cross-subject ERSP). For betweengroup analysis, we further computed the ERSP of Nonturners and Turners as well the 
1 difference ERSP for each cluster and computed the significance of these differences

2 using bootstrapping statistics $(\mathrm{p}<0.05)$.

3

4 Correlation analysis. In order to investigate functional aspects of brain dynamics during the navigation task, we correlated IC spectral power in different frequency bands of each navigation segments with the homing behavior. The baseline-corrected power values of the ICs of interest were sorted according to the adjusted homing angles in each trial. Power values and homing angles of every 5 trials were average into bins. Subsequently Pearson correlations between homing angles and power values in specific frequencies were computed. Likewise, a second correlation was computed to investigate the relation between homing errors and spectral power.

\section{Results}

\section{Homing performance}

Participants' homing responses are displayed in Fig. 1(C) with mean expected homing angles for both Turners and Nonturners indicated as dotted and straight line, respectively. Responses for increasingly lateral end positions were associated with increasing homing angles. However, homing responses for Turners and Nonturners consistently differed indicating that both strategy groups used a distinct reference frame for their homing responses even though the environment provided clear allothetic information. Turners, using an egocentric reference frame, indicated homing directions into opposite directions as compared to homing responses of Nonturners, using an allocentric reference frame. Turning direction had no impact on homing accuracy $\left[\mathrm{F}(1,375)=0.560, p=0.455\right.$, eta $\left.^{2}=0.001\right]$ and thus left and right turning trials were merged for a 2x9 mixed model ANOVA ('strategy' x 'end Position'). This analysis revealed significant differences in homing error for Turner and Nonturner participants $\left[\mathrm{F}(1,359)=16.568, p<0.001\right.$ eta $\left.^{2}=0.030\right]$ and a significant interaction of strategy and end position $\left[\mathrm{F}(8,359)=19.053, p<0.001\right.$, eta $\left.^{2}=0.283\right]$. Nonturners were more accurate for less eccentric end positions $\left(18.4^{\circ}, 26.5^{\circ}\right.$, and $\left.33.6^{\circ}\right)$ while Turners were more accurate for higher eccentricities (above $45^{\circ}$ ).

\section{EEG dynamics}


Selected clusters of ICs are displayed in Fig. 2 showing equivalent dipole locations projected onto the MNI brain with the corresponding scalp map for each cluster. Mean ERSP images (Fig. 3) display significant $(\mathrm{p}<0.05)$ phasic spectral power changes during the path segments compared to the control condition.

$* * * * * * * * * * * *$ insert Figure 2 here

Anterior Cingulate Cortex Cluster. Prominent spectral perturbations in the theta (4$7 \mathrm{~Hz}$ ) band were accompanied by power increase in thebeta (14-30Hz) and gamma band $(>31 \mathrm{~Hz})$ in or near the anterior cingulate cortex (Cls 1) during most path segments (shown in Fig. 3B). Strong power increases in the theta band were evident for both Turners and Nonturners. In line with previous research (Bischof and Boulanger, 2003; Jaiswal et al., 2010), theta modulation in the anterior cingulate cortex was increased during critical phases of the navigation task. Turners exhibited significantly stronger theta activity compared to Nonturners and the difference between the strategy groups was significant as evident from the difference plots (Fig. 3B most right column). Besides theta, beta power modulations were observed for both groups during navigation in the same cluster but without strategy-specific differences. Finally, significant power modulations in the gamma range were observed for Turners only resulting in significant differences between strategy groups.

$* * * * * * * * * * * *$ insert Figure 3 here $* * * * * * * * * * * *$

Motor Cortex Clusters. Alpha, and beta frequency bands demonstrated significant power modulations during navigation in or near left and right motor cortices (Fig. $3 \mathrm{C}$ and D). While bursts of increased low frequency power were observed accompanying specific aspects of the paths, i.e. acceleration and deceleration at starting and turning points, alpha power modulations were observed over the complete navigation period. The left and right motor cortex demonstrated increased desynchronization in the alpha frequency band $(8-13 \mathrm{~Hz})$ with different foci for the two strategy groups. Egocentric navigators revealed power modulations in the lower alpha band (8-10 Hz) while allocentric navigators demonstrated power modulations in the higher alpha band (10-13 Hz). This strategy-specific difference in left motor cortex was significant. In addition, egocentric navigators revealed stronger synchronization in the high alpha/low beta (12- 
$1 \quad 14 \mathrm{~Hz}$ ) and the high beta band (20-30 Hz) whereas allocentric navigators did not

2 demonstrate synchronizations in higher frequency bands. This resulted in additional significant differences in the beta band. For the right motor cluster, the strongest differences were observed for the $12-14 \mathrm{~Hz}$ frequency band with significantly stronger desynchronization for allocentric navigators.

Parietal Cortex Clusters. Both Turners and Nonturners revealed a significant wideband power burst time-locked to the onset of movement and strong bursts in the low frequency band time-locked to acceleration and deceleration phases during navigation in or near bilateral parietal cortex (Fig. $3 \mathrm{E}$ and F). Desynchronization in the alpha band and in its first harmonic frequency band became prominent before the stimulus turn accompanied by low frequency bursts in both parietal clusters. In or near the right parietal cortex, allocentric participants revealed alpha power increases during the turning segment, while egocentric participants revealed an alpha power increase during straight segments before and after a turn supporting previous results of right-literalized brain activation in parietal cortex during spatial navigation (Jacobs et al., 2010; White et al., 2012). Strategy differences in spectral modulations in the left parietal cortex were significant with egocentric navigators revealing stronger alpha desynchronization in the left parietal cortex compared to allocentric navigators.

Retrosplenial Cortex Cluster. With onset of motion, strong wide-band power bursts were observed in or near the retrosplenial cortex followed by broadband alpha and beta desynchronization up to the deceleration phase before a stimulus turn. As can be seen in Fig. 3 G, shortly before and during the stimulus turn, strong higher alpha and beta synchronization was observed for both Turners and Nonturners. However, Nonturners using an allocentric reference frame revealed a significantly stronger alpha desynchronization as compared to Turners using an egocentric reference frame as indicated in the difference plot. In addition, the difference plots revealed bursts of increased power in the high alpha/low beta bands after the stimulus turn that became most prominent at the end of the passage only for allocentric participants.

Occipital Cortex Clusters. Alpha power (8-13 Hz) and it first harmonic band revealed significant power changes in or near occipital cortex for egocentric as well as allocentric participants (Fig. $3 \mathrm{H}$ and I). With onset of navigation, a wide band power burst was 
1 observed mirroring the wide band burst in parietal and retrosplenial cortex for both 2 strategies groups. During navigation alpha and its harmonic bands were significantly stronger suppressed for egocentric participants during straight segments before and after the turn. During the stimulus turn a stronger alpha synchronization set in for allocentric navigators. After the stimulus turn, alpha was again suppressed in or near bilateral occipital cortex (Fig. 3I).

\section{EEG Dynamics related to Homing Performance}

Time-frequency analyses of brain dynamics of independent component clusters revealed path integration to be associated with activity in a wide spread cortical network, with modulations in distinct frequency bands for both Turners and Nonturners during navigation. To further investigate the possible role of modulations in different frequency bands associated with the use of distinct reference frames during navigation, Pearson correlations between EEG power in different frequencies during navigation and homing behavior (adjustments and errors) after navigation were computed. To control for false positives multiple comparisons were corrected using FDR. EEG power in different frequency bands showed significant $(\mathrm{p}<0.05)$ correlations with homing performance in several brain regions including parietal, motor, retrosplenial and occipital cortex. In this study, the homing performance showed the strongest correlation with theta and alpha modulations and was most prominent in retrosplenial (Figure 4) and left motor cortex (Figure 5). Figure 4 displays correlations coefficients of EEG power changes in retrosplenial cortex with homing adjustments for each navigation phase for Nonturners, using an allocentric SRF. The significant correlation in retrosplenial cortex was found only for Nonturner participants.

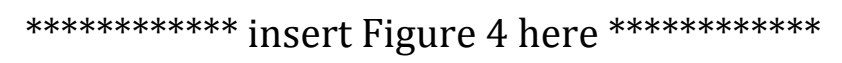
significant correlations with allocentric homing adjustments. A strong theta synchronization with onset of movement led to an overall positive correlation with homing adjustments. During later phases of the navigation task, theta modulations revealed a negative correlation with homing adjustments, i.e. more pronounced desynchronization in the frequency range between 4 and $7 \mathrm{~Hz}$ were observed for increasingly eccentric homing adjustments. Beside theta, the alpha frequency band 
1 demonstrated significant negative correlations with allocentric homing adjustments 2 throughout the navigation phase. Modulations in $12-14 \mathrm{~Hz}$ also revealed significant negative correlations with homing adjustments during navigation of the straight segments before and after the stimulus turn while no significant correlation was observed during the turn. The high alpha/low beta $(12-14 \mathrm{~Hz})$ modulation during the turn correlated positively with homing errors for Nonturners. The alpha, theta, and beta modulation remained significantly correlated to homing angles after eliminating the influence of homing errors by partial-correlations. While significant correlations of power modulations in RSC were observed for allocentric navigators, no such relationship was observed for Turners.

$* * * * * * * * * * * *$ insert Figure 5 here $* * * * * * * * * * * *$

Figure 5 displays correlations coefficients of alpha power changes in left motor cortex with homing adjustments during different navigation phases for participants using an allocentric or an egocentric SRF. A strong positive correlation of the alpha frequency band during the complete navigation period was observed for Nonturners. In other words, trials that were associated with larger homing errors revealed stronger alpha power in or near the left motor cortex. In contrast, negative correlations of the alpha frequency band were observed for Turners in or near the left motor cortex during the complete navigation period. The different correlation pattern for the strategy groups suggests that the self-motion information in motor cortex has different effects on the use of allocentric and egocentric SRF.

The correlations of alpha power and homing errors in the left motor cluster and the retrosplenial cluster were observed during similar navigation phases with higher correlations during the first straight segment compared to the following straight segments before the turn and the strongest correlations before the stimulus turn. In summary, EEG activities in or near retrosplenial cortex demonstrated a linear relationship to homing adjustment while EEG activities in or near motor cortex revealed a linear relationship with homing errors.

\section{Discussion}


Previous studies suggested a fronto-parietal network to subserve the computation

2 of allocentric and egocentric reference frames (Galati et al., 2000; Zaehle et al., 2007) with prefrontal areas and the ACC subserving diverse navigation-related functions such as working memory, attention, and route planning (Spiers and Maguire, 2006; Simons et al., 2008; González-Burgos et al., 2012). In the present study, a cluster in or near the ACC revealed significant theta band synchronization reflecting spatial working memory demands to compute and maintain participants' spatial representation for successful path integration. Strong increases in theta power shortly before and during stimulus turns may reflect increasing task demands associated with upcoming and actual heading changes. These findings replicate previous reports of increased theta power in frontal cortex during more demanding navigation periods (Kahana et al., 1999; Caplan et al., 2003). Turners demonstrated stronger theta activity compared to Nonturners. This is in line with more information that has to be updated based on an egocentric reference frame, including position and orientation changes with each rotation and translation, while an allocentric reference frame requires only updating of position but not orientation of the navigator (Klatzky, 1998). As a consequence, egocentric spatial updating of the self requires more working memory resources than allocentric spatial updating (Spiers, 2008).

The present study further demonstrated significant task-related alpha modulation in the parietal cortex. Modulation of alpha power in the parietal cortex has been previously observed during spatial learning and maintenance of orientation (Gramann et al., 2010; Plank et al., 2010; Snider et al., 2013). In the present study, alpha and the first harmonic were suppressed before stimulus turns in the right parietal region. This desynchronization might reflect an increase in spatial attention to integrate information based on perceived visual flow with a representation of the navigators' position. During the subsequent stimulus turn, Nonturners revealed significantly stronger alpha power compared to Turners. This suggests that the right parietal cortex might be less active during actual heading changes in allocentric navigators to allow integration of heading changes with allocentric representations. This is supported by studies observing stronger activation in the right parietal cortex in virtual navigation task in environments with clear allothetic information (Jacobs et al., 2010; White et al., 2012) and specific activation of right parietal structures for processing of allocentric spatial information and visual spatial attention (Zaehle et al., 2007; Shulman et al., 2010). Besides alpha modulation, low frequency modulations were observed in parietal areas. Theta bursts 
1 time locked to acceleration and deceleration phases of the movement revealed no

2 differences between Turners and Nonturners. These low frequency bursts likely reflect processing of spatial information related to self-motion irrespective of the spatial reference frame used for navigation. Task-related modulations in distinct frequency bands located in the parietal cortex point to functionally distinct roles (e.g., motion perception and visuo-spatial attention) of these bands for spatial navigation.

Besides the parietal cortex, the motor cortex demonstrated strong power modulation in the alpha frequency band. Alpha modulations in left motor cortex were negatively correlated with Turner's homing errors for nearly the complete navigation phase. Alpha desynchronization was more pronounced when Turners overestimated the homing angle and less pronounced when they underestimated the homing angle. Assuming alpha suppression to reflect motor cortex activity during real or imagined movement (Pfurtscheller and Klimesch, 1991), stronger simulation of rotation during stimulus turns could explain overestimation of homing angles. Less pronounced imagined rotations, in contrast, would lead to underestimations. In addition, correlations of alpha modulation with homing error were positive for allocentric navigators. This is a surprising finding since the associated simulated motor action is assumed to be less pronounced for Nonturners. Nonturners demonstrated accurate homing performance with low alpha changes in motor cortex. Stronger alpha power changes were accompanied by larger homing errors for trials with both under-and over-estimations. This implies that allocentric navigators perform best with a moderate input from the motor cortex. Stronger involvement of motor cortex as reflected in higher alpha desynchronization might indicate too much resources used for egocentric computations that interfere with the computation of an allocentric representation. In summary, while egocentric navigators seem to make use of information derived from motor cortex, for allocentric navigators an increasing reliance on this information seemed to conflict with the allocentric response mode.

Most prominent in the current study was the activity pattern in or near the RSC. Studies on spatial navigation demonstrated the involvement of the RSC in various kinds of navigation tasks (Maguire, 2001; Spiers and Maguire, 2006; Vann et al., 2009), especially in tasks requiring transformation of egocentric and allocentric information into alternative reference frames (Byrne et al., 2007; Vann et al., 2009; Zhang et al., 2012; Dhindsa et al., 2014). Previous studies also showed modulations of the alpha frequency band (8-13 Hz) during spatial navigation in or near RSC (Gramann et al., 2010). In the 
present study, both strategy groups demonstrated task-related alpha desynchronization

2 in the RSC during straight segments before and after the stimulus turn. In contrast to previous studies we also found alpha synchronization during stimulus turns for both

4 strategy groups. In the present study all stimulus turns had a constant angle of 90 degrees and rotations happened on the spot. While the stimulus turns were not central to compute changes in position, they still contained changes in visual flow based on allothetic spatial information. Thus, during all segments of a passage sufficient allothetic information in form of a structured environment was present and allowed to compute position and bearing from the start. Therefore we interpret alpha suppression in the RSC to be functionally equivalent with alpha suppression in other cortical areas like the parietal or occipital cortices in that it indicates activation of RSC to process incoming (spatial) information (Pfurtscheller and Lopes da Silva, 1999).

Alpha modulation in the RSC correlated with homing performance only in allocentric navigators revealing larger homing angles to be accompanied by stronger alpha suppression. Together with alpha suppression during straight segments in occipital cortex and low frequency synchronization in right-parietal cortex this pattern provides further evidence that alpha suppression in the RSC is central to computing and maintaining an allocentric positional representation by combining visual-flow information from occipital and parietal regions (Vann et al., 2009) with allothetic cues to map egocentric viewpoints onto an allocentric reference frame (Knight and Hayman, 2014). Our results support the notion that alpha desynchronization in RSC during straight segments reflects integration of egocentric self-motion with a constant allocentric heading representation and possibly other allocentric locational representations. In addition to the alpha (8-13 Hz) desynchronization, we found synchronizations of the high alpha/low beta (12-14 $\mathrm{Hz}$ ) frequency range during stimulus turns for both strategy groups. The stimulus turn in the present study involved on the spot rotations, unlike previous studies that also included translations during the turn. Recent results from Gomez and colleagues showed stronger RSC activation during rotation at a fixed position compare to a continuous movement (Gomez et al., 2014). Therefore, the shift of synchronization from the alpha band to a higher frequency may indicate a distinct underlying cognitive process for orientation changes.

Taken together, the task-related modulations of different frequencies in RSC imply two different modes of retrosplenial activity: One mode, reflected in alpha desynchronization, indicates the continuous integration of ego-motion with allocentric 
1 reference frames (e.g., with respect to environmental boundaries). The RSC receives

2 information on ego-motion from occipital and parietal cortices processing visual flow changes (Vann et al., 2009; Knight and Hayman, 2014). This information is integrated with allocentric reference frames to provide information on changes in position and orientation with respect to the initial position and heading direction as long as the navigator moves in the environment or whenever the current position and orientation has to be retrieved for further computations. A second mode, reflected in modulations of the higher alpha/lower beta band (12-14 Hz), subserves the computation and maintenance of allocentric heading itself (Byrne et al., 2007; Gomez et al., 2014). This is most pronounced during actual heading changes but becomes necessary whenever allocentric heading is used to align information coded in an egocentric SRF (e.g., visual flow) that is misaligned with the allocentric heading representation. In the present task, the second mode would be present during the turning segment and after the turn when egocentric visual flow was misaligned with the initial reference direction.

In conclusion, differences in reference frame proclivity were replicated in this new task, using a clearly structured environment with allothetic information. Thus, individual reference frame proclivities are not easily influenced by allothetic information and might indicate more general differences in spatial abilities (Gramann, 2013). Successful navigation was associated with activity in a wide cortical network. Egocentric navigators showed significantly stronger theta power increases in the medial frontal cortex and beta increases in motor cortex. Nonturners, in contrast, demonstrated significantly stronger alpha modulation in the RSC, parietal, and occipital cortex. Alpha oscillation in the RSC and motor cortex of allocentric navigators revealed significant correlations with homing angles and homing errors, respectively. The results support the claim that the retrosplenial cortex is central to computing heading and translating egocentric and allocentric spatial information into different reference frames. Modulations in the alpha and the high alpha/low beta band with different time courses in RSC further provide first evidence of these two distinct neural processes reflecting translation of spatial information based on distinct reference frames and computation and maintenance of heading changes, respectively.

\section{Acknowledgments}

This work was supported in part by the UST-UCSD International Center of Excellence in Advanced Bio-engineering sponsored by the Taiwan National Science Council I-RiCE 
1 Program under Grant Number: MOST 103-2911-I-009-101, in part by MOST 103-26272 E-009-001, in part by the Aiming for the Top University Plan, under Contract: 104W963, 3 and in part by the Army Research Laboratory: W911NF-10-2-0022. 


\section{Figure Legends}

2 Figure 1: Experiment design and behavior results. (A) The virtual maze was a grid-like

3 navigation environment composed by 7 columns and 5 rows displayed as road. For each

4 stonewall structure displayed at the intersection of the grid, the squares in the underlying grid were randomly eliminated with a probability of 0.8 (showed as the dash squares). The irregularities in these wall structures were created to increase the complexity of the environment. Participants started form the homing position (star symbol) following the guiding arrow to one of the destination (the crossroad marked as a-i and $\underline{a}-\underline{i}$ ). (B) Screenshots of homing task and control task. The homing task required subject to keep their orientation during the navigation phase, 6-14 sec depending on the path length until the end position was reached. The control task provided about 60 seconds of visual flow during random walk through the same environment. (C) Circular mean homing responses for each participant for destination a-i and $\underline{a}-\underline{i}$. Solid gray lines and bold grey line indicate responses and mean response for Nonturners, respectively. Dotted lines and bold dotted line indicate responses and mean response for Turners, respectively. Circular markers display expected homing angles for Turners and Nonturners. (D) Illustration of homing responses of Nonturners (dark grey head) and Turners (light grey head) at the end of a passage. For a path with one turn (to the right in this figure) the homing directions differ for Turners and Nonturners with the former strategy group pointing back and to their right and the later strategy group pointing back and to their left.

Figure 2: Dipole locations of independent component clusters and respective mean scalp maps. The middle row displays equivalent dipole models of each independent component (with small spheres) and the centroids of each component cluster (big spheres) projected onto the standard brain. The average scalp map of each cluster is displayed and color-coded corresponding to the color-coding used for the dipoles models. For each cluster the number of participants and the number of ICs is given. Cluster centroids are located in or near the anterior frontal cortex (Cls 1), left motor cortex (Cls 2), right motor cortex (Cls 3), left posterior parietal cortex (Cls 4), right posterior parietal cortex (Cls 6), retrosplenial cortex (Cls 6), occipital cortex (cls 7, 8).

Figure 3: Event-related spectral perturbation (ERSP) of brain dynamics for selected 
1 clusters. (A) Top row of screen shots displays different segments of a passage for both egocentric and allocentric participants. Straight: following the guiding arrow to move forward. Deceleration: approaching the turn and slowing down to prepare for turning.

Figure 4: A) Screen shots displaying different segments of a passage. B) ERSP of allocentric participants with cluster ICs located in or near the RSC. Color-coding as in figure 3. C-E) Correlation coefficients computed between event-related spectral perturbations (ERSP) and homing adjustments for allocentric participants in theta (C), alpha (D), and the high alpha/low beta (E) frequency band. (F) Correlation coefficients computed between homing error and power changes for allocentric participants in the low beta frequency band. *: p<0.05, ${ }^{* *}$ : $\mathrm{p}<0.01$ (all p FDR corrected).

Figure 5: Correlation coefficients computed between event-related spectral perturbations (ERSP) and homing errors of allocentric and egocentric participants for the left motor cluster. (A) Screen shots displaying different segments of a passage. (B) ERSP of allocentric and egocentric participants for the cluster with its centroid located in or near the left motor cluster. Color-coding as in figure 3. Correlation plot of homing error and power changes of in alpha frequency band as showed for Nonturners (C) and Turners (D). *: p<0.05, **: p<0.01 (all FDR corrected). 
Bischof, W.F., Boulanger, P., 2003. Spatial navigation in virtual reality environments: an EEG analysis. Cyberpsychol. Behav. 6, 487-495.

Bohbot, V.D., Lerch, J., Thorndycraft, B., Iaria, G., Zijdenbos, A.P., 2007. Gray matter differences correlate with spontaneous strategies in a human virtual navigation task. J. Neurosci. 27, 10078-10083.

Bremmer, F., Schlack, A., Shah, N.J., Zafiris, O., Kubischik, M., Hoffmann, K.P., Zilles, K., Fink, G.R., 2001. Polymodal motion processing in posterior parietal and premotor cortex: A human fMRI study strongly implies equivalencies between humans and monkeys. Neuron 29, 287-296.

Burgess, N., 2006. Spatial memory: how egocentric and allocentric combine. Trends Cogn. Sci. 10, 551-557.

Byrne, P., Becker, S., Burgess, N., 2007. Remembering the past and imagining the future: a neural model of spatial memory and imagery. Psychol. Rev. 114, 340-375.

Caplan, J.B., Madsen, J.R., Schulze-Bonhage, A., Aschenbrenner-Scheibe, R., Newman, E.L., Kahana, M.J., 2003. Human theta oscillations related to sensorimotor integration and spatial learning. J. Neurosci. 23, 4726-4736.

Chiu, T.-C., Gramann, K., Ko, L.-W., Duann, J.-R., Jung, T.-P., Lin, C.-T., 2012. Alpha modulation in parietal and retrosplenial cortex correlates with navigation performance. Psychophysiology 49, 43-55.

Cohen, Y.E., Andersen, R.A., 2002. A common reference frame for movement plans in the posterior parietal cortex. Nat. Rev. Neurosci. 3, 553-562.

Delorme, A., Makeig, S., 2004. EEGLAB: An open source toolbox for analysis of single-trial EEG dynamics including independent component analysis. J. Neurosci. Methods 134, 9-21.

Delorme, A., Palmer, J., Onton, J., Oostenveld, R., Makeig, S., 2012. Independent EEG sources are dipolar. PLoS One 7, e30135.

Dhindsa, K., Drobinin, V., King, J., Hall, G.B., Burgess, N., Becker, S., 2014. Examining the role of the temporo-parietal network in memory, imagery, and viewpoint transformations. Front. Hum. Neurosci. 8, 709.

Doeller, C.F., Barry, C., Burgess, N., 2010. Evidence for grid cells in a human memory network. Nature 463, 657-661.

Ekstrom, A.D., Kahana, M.J., Caplan, J.B., Fields, T.A., Isham, E.A., Newman, E.L., Fried, I., 2003. Cellular networks underlying human spatial navigation. Nature 425, 184-188. 
Galati, G., Lobel, E., Vallar, G., Berthoz, a, Pizzamiglio, L., Le Bihan, D., 2000. The neural basis of egocentric and allocentric coding of space in humans: a functional magnetic resonance study. Exp. Brain Res. 133, 156-64.

Goeke, C.M., König, P., Gramann, K., 2013. Different strategies for spatial updating in yaw and pitch path integration. Front. Behav. Neurosci. 7, 5.

Gomez, A., Cerles, M., Rousset, S., Rémy, C., Baciu, M., 2014. Differential hippocampal and retrosplenial involvement in egocentric-updating, rotation, and allocentric processing during online spatial encoding: an fMRI study. Front. Hum. Neurosci. 8, 150.

González-Burgos, I., Fletes-Vargas, G., González-Tapia, D., González-Ramírez, M.M., Rivera-Cervantes, M.C., Martínez-Degollado, M., 2012. Prefrontal serotonin depletion impairs egocentric, but not allocentric working memory in rats. Neurosci. Res. 73, 321-327.

Gramann, K., 2013. Embodiment of spatial reference frames and individual differences in reference frame proclivity. Spat. Cogn. Comput. 13, 1-25.

Gramann, K., Müller, H.J., Eick, E.-M., Schönebeck, B., 2005. Evidence of separable spatial representations in a virtual navigation task. J. Exp. Psychol. Hum. Percept. Perform. 31, 1199-1223.

Gramann, K., Onton, J., Riccobon, D., Mueller, H.J., Bardins, S., Makeig, S., 2010. Human brain dynamics accompanying use of egocentric and allocentric reference frames during navigation. J. Cogn. Neurosci. 22, 2836-2849.

Gramann, K., Wing, S., Jung, T.-P., Viirre, E., Riecke, B.E., 2012. Switching Spatial Reference Frames for Yaw and Pitch Navigation. Spat. Cogn. Comput. 12, 159-194.

Howard, L.R., Javadi, A.H., Yu, Y., Mill, R.D., Morrison, L.C., Knight, R., Loftus, M.M., Staskute, L., Spiers, H.J., 2014. The hippocampus and entorhinal cortex encode the path and euclidean distances to goals during navigation. Curr. Biol. 24, 1331-1340.

Iaria, G., Petrides, M., Dagher, A., Pike, B., Bohbot, V.D., 2003. Cognitive Strategies Dependent on the Hippocampus and Caudate Nucleus in Human Navigation: Variability and Change with Practice. J. Neurosci. 23, 5945-5952.

Iglói, K., Zaoui, M., Berthoz, A., Rondi-Reig, L., 2009. Sequential egocentric strategy is acquired as early as allocentric strategy: Parallel acquisition of these two navigation strategies. Hippocampus 19, 1199-1211.

Jacobs, J., Korolev, I.O., Caplan, J.B., Ekstrom, A.D., Litt, B., Baltuch, G., Fried, I., SchulzeBonhage, A., Madsen, J.R., Kahana, M.J., 2010. Right-lateralized brain oscillations in human spatial navigation. J. Cogn. Neurosci. 22, 824-836.

Jacobs, J., Weidemann, C.T., Miller, J.F., Solway, A., Burke, J.F., Wei, X.-X., Suthana, N., Sperling, M.R., Sharan, A.D., Fried, I., Kahana, M.J., 2013. Direct recordings of gridlike neuronal activity in human spatial navigation. Nat. Neurosci. 16, 1188-1190. 
Jaiswal, N., Ray, W., Slobounov, S., 2010. Encoding of visual-spatial information in working memory requires more cerebral efforts than retrieval: Evidence from an EEG and virtual reality study. Brain Res. 1347, 80-89.

Janzen, G., van Turennout, M., 2004. Selective neural representation of objects relevant for navigation. Nat. Neurosci. 7, 673-677.

Just, M.A., Carpenter, P.A., 1985. Cognitive coordinate systems: accounts of mental rotation and individual differences in spatial ability. Psychol. Rev. 92, 137-172.

Kahana, M.J., Sekuler, R., Caplan, J.B., Kirschen, M., Madsen, J.R., 1999. Human theta oscillations exhibit task dependence during virtual maze navigation. Nature 399, 781-784.

Kelly, J., McNamara, T., Bodenheimer, B., 2008. The shape of human navigation: How environmental geometry is used in maintenance of spatial orientation. Cognition $109,281-286$.

Klatzky, R.L., 1998. Allocentric and egocentric spatial representations: definitions, distinctions, and interconnections. In: Freska, C., Havbel, C. (Eds.), Spatial Cognition. : An Interdisciplinary Approach to Representing and Processing Spatial Knowledge. Springer, Heidelberg, pp. 1-17.

Knight, R., Hayman, R., 2014. Allocentric directional processing in the rodent and human retrosplenial cortex. Front. Hum. Neurosci. 8, 135.

Maguire, E.A., 2001. The retrosplenial contribution to human navigation: a review of lesion and neuroimaging findings. Scand. J. Psychol. 42, 225-238.

Maguire, E.A., Burgess, N., Donnett, J.G., Frackowiak, R.S., Frith, C.D., O’Keefe, J., 1998. Knowing where and getting there: a human navigation network. Science 280, 921924.

Makeig, S., 1993. Auditory event-related dynamics of the EEG spectrum and effects of exposure to tones. Electroencephalogr. Clin. Neurophysiol. 86, 283-293.

Marchette, S.A., Vass, L.K., Ryan, J., Epstein, R.A., 2014. Anchoring the neural compass: coding of local spatial reference frames in human medial parietal lobe. Nat. Neurosci. 17, 1598-1606.

Oostenveld, R., Oostendorp, T.F., 2002. Validating the boundary element method for forward and inverse EEG computations in the presence of a hole in the skull. Hum. Brain Mapp. 17, 179-192.

Palmer, J.A., Kreutz-Delgado, K., Makeig, S., 2006. Super-Gaussian mixture source model for ICA. In: Rosca, J., Erdogmus, D., Principe, J.C., Haykin, S. (Eds.), Lecture Notes in Computer Science. Springer, Berlin, pp. 854-861. 
Pfurtscheller, G., Klimesch, W., 1991. Event-related desynchronization during motor behavior and visual information processing. Electroencephalogr. Clin. Neurophysiol. Suppl. 42, 58-65.

Pfurtscheller, G., Lopes da Silva, F.H., 1999. Event-related EEG/MEG synchronization and desynchronization: basic principles. Clin. Neurophysiol. 110, 1842-57.

Plank, M., Müller, H., Onton, J., Makeig, S., Gramann, K., 2010. Human EEG correlates of spatial navigation within egocentric and allocentric reference frames. In: Hölscher, C., Shipley, T.F., Olivetti Belardinelli, M., Bateman, J.A., Newcombe, N.S. (Eds.), Spatial Cognition VII. Springer, Berlin, pp. 191-206.

Restat, J.D., Steck, S.D., Mochnatzki, H.F., Mallot, H.A., 2004. Geographical slant facilitates navigation and orientation in virtual environments. Perception 33, 667-687.

Riecke, B.E., 2008. Consistent Left-Right Reversals for Visual Path Integration in Virtual Reality: More than a Failure to Update One's Heading? Presence Teleoperators Virtual Environ. 17, 143-175.

Riecke, B.E., Wiener, J.M., 2007. Can People Not Tell Left from Right in VR? Point-to-origin Studies Revealed Qualitative Errors in Visual Path Integration. In: 2007 IEEE Virtual Reality Conference. IEEE, pp. 3-10.

Seubert, J., Humphreys, G. W., Müller, H. J., \& Gramann, K. (2008). Straight after the turn: The role of the parietal lobes in egocentric space processing. Neurocase, 14(2), 204219.

Shulman, G.L., Pope, D.L.W., Astafiev, S. V, McAvoy, M.P., Snyder, A.Z., Corbetta, M., 2010. Right hemisphere dominance during spatial selective attention and target detection occurs outside the dorsal frontoparietal network. J. Neurosci. 30, 3640-3651.

Simons, J.S., Henson, R.N.A., Gilbert, S.J., Fletcher, P.C., 2008. Separable forms of reality monitoring supported by anterior prefrontal cortex. J. Cogn. Neurosci. 20, 447-457.

Spiers, H.J., 2008. Keeping the goal in mind: Prefrontal contributions to spatial navigation. Neuropsychologia 46, 2106-2108.

Snider, J., Plank, M., Lynch, G., Halgren, E., Poizner, H., 2013. Human cortical $\theta$ during free exploration encodes space and predicts subsequent memory. J. Neurosci. 33, 15056-15068.

Spiers, H.J., 2008. Keeping the goal in mind: Prefrontal contributions to spatial navigation. Neuropsychologia 46, 2106-2108.

Spiers, H.J., Maguire, E.A., 2006. Thoughts, behaviour, and brain dynamics during navigation in the real world. Neuroimage 31, 1826-1840. 
Touretzky, D.S., Redish, A.D., 1996. Theory of rodent navigation based on interacting representations of space. Hippocampus 6, 247-270.

Vann, S.D., Aggleton, J.P., Maguire, E.A., 2009. What does the retrosplenial cortex do? Nat. Rev. Neurosci. 10, 792-802.

White, D.J., Congedo, M., Ciorciari, J., Silberstein, R.B., 2012. Brain oscillatory activity during spatial navigation: theta and gamma activity link medial temporal and parietal regions. J. Cogn. Neurosci. 24, 686-697.

Wolbers, T., Büchel, C., 2005. Dissociable retrosplenial and hippocampal contributions to successful formation of survey representations. J. Neurosci. 25, 3333-3340.

Wolbers, T., Weiller, C., Büchel, C., 2004. Neural foundations of emerging route knowledge in complex spatial environments. Brain Res. Cogn. Brain Res. 21, 401411.

Wolbers, T., Wiener, J.M., Mallot, H.A., Büchel, C., 2007. Differential recruitment of the hippocampus, medial prefrontal cortex, and the human motion complex during path integration in humans. J. Neurosci. 27, 9408-9416.

Zaehle, T., Jordan, K., Wüstenberg, T., Baudewig, J., Dechent, P., Mast, F.W., 2007. The neural basis of the egocentric and allocentric spatial frame of reference. Brain Res. 1137, 92-103.

Zhang, H., Copara, M., Ekstrom, A.D., 2012. Differential recruitment of brain networks following route and cartographic map learning of spatial environments. PLoS One 7, e44886. 